\title{
Treatment of clinically isolated syndrome with low dose naltrexone
}

\author{
M. Ludwig ${ }^{1}$, A.P. Turel ${ }^{2}$, I.S. Zagon ${ }^{1}$ and P.J. McLaughlin ${ }^{1 *}$ \\ ${ }^{1}$ Department of Neural and Behavioral Sciences, Pennsylvania State University College of Medicine, Hershey, USA \\ ${ }^{2}$ Department of Neurology Pennsylvania State University College of Medicine, Hershey, USA
}

\begin{abstract}
Clinically isolated syndrome (CIS) is an early sign of multiple sclerosis (MS). Despite being difficult to diagnose, the presentation of CIS is often accompanied by immunotherapy that is expensive and may have side effects. The purpose of this study was to evaluate CIS patients prescribed low dose naltrexone (LDN), an inexpensive, safe and tolerable compound that triggers endogenous enkephalin/endorphin release by the patient. LDN was prescribed to four female patients diagnosed with CIS in addition to FDA approved therapy. Retrospective chart reviews beginning in 2011 until present document that these women did not transition to clinically definite MS over a period of 4 years. Moreover, the patients reported that LDN therapy was without side effect and stabilized or improved their perceived quality of life and clinical status, including the reduction of fatigue. Thus, LDN may provide a safe and inexpensive alternative or addition to standard therapy of CIS.
\end{abstract}

\section{Introduction}

Clinically isolated syndrome (CIS) as defined by the $2010 \mathrm{McD}$ onald Criteria describes the first signs of neurologic symptoms that may evolve into multiple sclerosis (MS) [1,2]. Typically, the symptoms last a day or two and are associated with inflammation in the central nervous system (CNS). Monofocal CIS is ascribed to an experience related to a single symptom such as optic neuritis. Multifocal CIS involves two or more neurologic symptoms such as optic neuritis and numbness or tingling in one extremity. Transition to clinically definite MS requires a second neurological episode with new or worsening lesions of the brain or spinal cord as confirmed by MRI. Patients with CIS do not always progress to clinically definite MS, and the second attack may occur months and years later. The initial diagnosis of CIS is important because patients are encouraged to begin some form of treatment to delay the onset of MS.

Several medications are FDA approved for CIS including Avonex ${ }^{\circ}$, Betaseron, and Copaxone ${ }^{\circ}$. These therapies are expensive, may require injections, and often have intolerable side-effects [1]. A novel biotherapeutic used alone and in combination with these diseasemodifying therapies (DMT) is oral low dose naltrexone (LDN). Reports from clinical trials on LDN $[3,4]$, as well as retrospective chart reviews [5] have demonstrated few or no confounding effects with LDN when taken alone or in combination with DMT. Subjective feedback from patients on LDN, in some cases for more than 500 days, revealed that patients had perceived reductions in fatigue and disease progression. Both clinical trials and chart reviews confirmed that LDN was safe and well tolerated.

The mechanism of action of LDN is that low dosages of the opioid antagonist block the interfacing of endogenous peptides and their receptors for a short period of time, producing a biofeedback that upregulates the secretion of enkephalins and endorphins, as well as related receptors, thus allowing for enhanced interaction of the inhibitory peptide $\left[\mathrm{Met}^{5}\right]$-enkephalin (i.e., opioid growth factor, OGF) [5-8]. LDN is recommended at dosages of 3-4.5 $\mathrm{mg}$ per day, with at least $24 \mathrm{hr}$ between oral consumption. The duration of opioid receptor blockade is critical for the efficacy of LDN, and receptors can only be blocked 4-6 hr daily [8].

Whether LDN could be used as an inexpensive (less than $\$ 50$ per month), safe and tolerable treatment for patients with CIS is unclear. This report focuses on the progression of disease for four female patients diagnosed with CIS. Following full explanation and consent, all patients were offered the FDA approved agents for CIS and/or LDN at the time of diagnosis. Patients $\mathrm{A}$ and $\mathrm{B}$ received LDN following the diagnosis of CIS in addition to glatiramer acetate, whereas patients $\mathrm{C}$ and D began LDN therapy following the diagnosis of CIS with no other disease modifying therapy.

\section{Patient histories}

\section{Case 1}

Patient $\mathrm{A}$ is a 30 -year-old married female who came to the clinic in late 2011 with complaints of blurred vision. She used alcohol occasionally, but did not use tobacco. A cervical spinal MRI and physical examination were normal. A brain MRI revealed multiple ovoid periventricular lesions suggestive of demyelinating disease. The neurological examination revealed minimal pallor of the right optic disk. The remainder of her examination was normal. The optic neuritis resulted in the diagnosis of CIS. The patient began treatment with 20 $\mathrm{mg}$ glatiramer acetate and $3 \mathrm{mg}$ naltrexone daily.

Correspondence to: Dr. P.J. McLaughlin, Department of Neural \& Behavioral Sciences, MC H109, Penn State University College of Medicine, 500 University Drive, Hershey, PA 17033 USA, E-mail: pxm9@psu.edu

Key words: clinically isolated syndrome, low dose naltrexone, multiple sclerosis, disease progression

Received: July 28, 2015; Accepted: September 03, 2015; Published: September 07,2015 


\section{Case 2}

Patient B is a 36-year-old female who presented to the clinic in late 2011 with complaints of left arm and leg weakness. She was married and had no history of tobacco or alcohol use. The MRI of the patient's cervical spinal cord was normal. A brain MRI showed one periventricular lesion in the right hemisphere. CSF analysis was normal. The general physical examination was normal. The neurological exam revealed mild left-sided weakness with increased motor tone. After 3 months, the patient began treatment with $20 \mathrm{mg}$ glatiramer acetate and after 6 months, the patient added $3 \mathrm{mg}$ naltrexone daily.

\section{Case 3}

Patient $\mathrm{C}$ is a 50 -year-old married female that presented to the clinic in mid 2011 with complaints of occasional blurred vision in her right eye. She also complained of fatigue and often felt off balance. This patient had no history of tobacco or alcohol use. The patient did have a sibling with a history of MS. The brain MRI revealed non-specific punctuate periventricular lesions. The cervical spinal cord MRI was normal, as was her physical examination. The patient's neurological examination revealed mild optic atrophy of the medial right optic disk. The patient began $3 \mathrm{mg}$ naltrexone daily. After 3 months, the patient requested a DMT, and interferon- $\beta 1$ a (Avonex) was prescribed in addition to the LDN.

\section{Case 4}

Patient D is a 42-year-old female presenting to the clinic in early 2011 with complaints of blurred vision and diplopia. She is single, has no tobacco usage, and drank a glass or two of wine per week. The patient underwent a brain MRI, which revealed several small nonspecific subcortical lesions. General physical examination was normal, and the patient was able to complete a timed $25 \mathrm{ft}$ walk in 4.33 and 4.22 seconds. The neurological exam was essentially normal, with the exception of mild pallor in the left optic nerve. At that time, the patient was diagnosed with CIS, declined the standard approved therapies for CIS, and she began $3 \mathrm{mg}$ naltrexone daily. In a clinic visit three months later, CSF analysis was obtained. This analysis revealed greater than 5 oligoclonal bands, elevated IgG synthesis, and showed no evidence of viral meningitis. The CSF cell count was normal. This CSF analysis put her at higher risk for developing clinically definite multiple sclerosis. The patient began $20 \mathrm{mg}$ glatiramer acetate daily. Later, due to changes in the McDonald diagnostic criteria and changes in follow-up providers, Patient D was diagnosed with relapsing remitting multiple sclerosis (RRMS) in late 2012.

\section{Discussion}

All four patients were on LDN therapy for several years, with patients $\mathrm{A}, \mathrm{B}$, and $\mathrm{C}$ not progressing to clinically definite MS within a 4-year period (at least through April 2015). Patients A and B, who began $3 \mathrm{mg}$ naltrexone therapy along with glatiramer acetate therapy remained stable without side effects attributed to naltrexone therapy. Patient A noted improvement in prior symptoms of fatigue and a stabilized neurologic status. She continues on both glatiramer acetate and LDN therapy. Patient B reported neither side effects nor apparent benefits to fatigue levels from naltrexone therapy, and has remained clinically stable. This patient discontinued LDN therapy in late 2014 when her prescription expired. Patient $\mathrm{C}$ continues to remain stable and subsequent brain MRIs were stable. This patient reported that LDN improved her perceived fatigue levels and quality of life. Patient $D$ reported a side effect of insomnia. She also reported improvement on her perceived fatigue and improvement in her quality of life, LDN kept her disease stable, despite her diagnosis shifting from CIS to RRMS. However she has recently discontinued her naltrexone therapy when the prescription expired. The patient's most recent MRI showed slight increase in white matter foci over her previous, improved MRI.

These data correlate well with those from larger clinical studies such as the PreCISe study [9] and BENEFIT study [10] reporting that $25 \%$ and $28 \%$ of patients taking glatiramer acetate or Betaseron, respectively, transitioned to clinically definite MS in comparison to placebo controls. Following the initial study with Betaseron, patients were offered to continue on interferon $\beta$. The former placebo group was then considered the delayed treatment group, and the group that continued treatment with interferon $\beta$ was then the considered the early treatment group. At the end of the three year follow-up study, 37\% of the early treatment group had progressed onto MS, while $51 \%$ of the delayed treatment group had progressed [10]. The early treatment with interferon beta reduced the risk of progression to MS by $41 \%$ at the end of three years, and $37 \%$ after five years [11].

The present study revealed that 4 patients receiving LDN at the time of CIS diagnosis did not transition to clinically definite MS over a period of several years. The data support patient-reported observations indicating that LDN stabilized or increased the quality of life without significant effects, reduced fatigue, and was perceived to improve clinical status.

These data corroborate and extend a recent retrospective chart review that reported on tolerability and safety of low doses of naltrexone in patients with clinically definite MS [12]. Patients 18 years of age or over who were diagnosed with MS were offered $3 \mathrm{mg}$ naltrexone, as a complementary therapy. Self-reported indicators suggested that most patients had an improved perception of less fatigue, and reported minimal side effects. The side effects associated with low doses of naltrexone included mild insomnia, vivid dreams, and/or rash, but were tolerable in comparison to the adverse effects such as flu-like symptoms, gastrointestinal, injection site reactions, depression, and in severe cases thoughts of suicide, anaphylaxis and seizure [13].

Thus, preclinical studies showing the efficacy of LDN in mouse models of experimental autoimmune encephalomyelitis $[7,14-16]$, as well as retrospective chart reviews of patients with clinically defined MS indicating safety and tolerability of LDN [12], and the current evidence that patients with CIS do not progress to defined MS any faster than those on DMT support that LDN therapy may provide a safe, inexpensive, and tolerable alternative or additive for patients diagnosed with CIS.

\section{Informed consent}

Informed consent was not required for this retrospective study.

\section{Authorship and contributorship}

All authors made significant contributions to the design of study, data collection, interpretation, and/or writing of the manuscript. All authors have read and approve the contents of the final manuscript. ML completed this project as partial fulfillment of his doctoral studies at Pennsylvania State University; his advisor, PJM is the corresponding author.

\section{Acknowledgements}

This work was supported in part by The Shockey Family Foundation. 


\section{Conflict of interest}

There are no conflicts of interest for any author.

\section{References}

1. Clinically isolated syndrome (CIS) http://national mssociety.org. Website accessed July 2015.

2. Polman CH, Reingold SC, Banwell B, Clanet M, Cohen JA, et al. (2011) Diagnostic criteria for multiple sclerosis: 2010 revisions to the McDonald criteria. Ann Neurol 69: 292-302. [Crossref]

3. Sharafaddinzadeh N, Moghtaderi A, Kashipazha D, Majdinasab N, Shalbafan B (2010) The effect of low-dose naltrexone on quality of life of patients with multiple sclerosis: a randomized placebo-controlled trial. Mult Scler 16: 964-969. [Crossref]

4. Cree BA, Kornyeyeva E, Goodin DS (2010) Pilot trial of low-dose naltrexone and quality of life in multiple sclerosis. Ann Neurol 68: 145-150.[Crossref]

5. Donahue RN, McLaughlin PJ, Zagon IS (2011) Low-dose naltrexone targets the opioid growth factor-opioid growth factor receptor pathway to inhibit cell proliferation: mechanistic evidence from a tissue culture model. Exp Biol Med 236:1036-1050. [Crossref]

6. Zagon IS, McLaughlin PJ (2014) Endogenous opioids and the treatment of multiple sclerosis. J Neurol Neurophysiol S12.

7. Hammer LA, Zagon IS, McLaughlin PJ (2015) Low dose naltrexone treatment of established relapsing-remitting experimental autoimmune encephalomyelitis. $J$ Mult Scler 2:2

8. McLaughlin PJ, Zagon IS (2015) Duration of opioid receptor blockade determines biotherapeutic response. Biochem Pharmacol. [Crossref]
9. Comi G, Martinelli V, Rodegher M et al. (2009) Effect of glatiramer acetate on conversion to clinically definite multiple sclerosis in patients with clinically isolated syndrome (PreCISe study): a radomised, double-blind, placebo-controlled trial. Lancet 374:1503-1511. [Crossref]

10. Kappos L, Freedman MS, Polman CH, Edan G, Hartung HP, et al. (2007) Effect of early versus delayed interferon beta- $1 \mathrm{~b}$ treatment on disability after a first clinical event suggestive of multiple sclerosis: a 3-year follow-up analysis of the BENEFIT study. Lancet 370: 389-397. [Crossref]

11. Kappos L, Freedman MS, Polman CH, Edan G, Hartung HP, et al. (2009) Long-term effect of early treatment with interferon beta- $1 \mathrm{~b}$ after a first clinical event suggestive of multiple sclerosis: 5-year active treatment extension of the phase 3 BENEFIT trial. Lancet-Neurol 8: 987-997. [Crossref]

12. Turel AP, Oh KH, Zagon IS, McLaughlin PJ (2015) Low Dose Naltrexone for Treatment of Multiple Sclerosis: A Retrospective Chart Review of Safety and Tolerability. J Clin Psychopharmacol 35: 609-611. [Crossref]

13. Kennedy PM (2013) Impact of delayed diagnosis and treatment in clinically isolated syndrome and multiple sclerosis. J Neurosci Nurs 45: S3-13. [Crossref]

14. RahnKA, McLaughlin PJ, Zagon IS (2011) Prevention and diminished expression of experimental autoimmune encephalomyelitis by low dose naltrexone (LDN) or opioid growth facor (OGF) for an extended period: Therapeutic implications for multiple sclerosis. Brain Res 1381: 243-253. [Crossref]

15. Hammer LA, Zagon IS, McLaughlin PJ(2015) Low dose naltrexone treatment of established relapsing-remitting experimental autoimmune encephalomyelitis. $J$ Multi Scler 2: 136 .

16. Hammer LA, Waldner H, Zagon IS, McLaughlin PJ (2015) Opioid growth factor and low-dose naltrexone impair central nervous system infiltration by CD4 + T lymphocytes in established experimental autoimmune encephalomyelitis, a model of multiple sclerosis. Exp Biol Med (Maywood). [Crossref]

Copyright: $\odot 2015$ Ludwig M. This is an open-access article distributed under the terms of the Creative Commons Attribution License, which permits unrestricted use, distribution, and reproduction in any medium, provided the original author and source are credited. 